\title{
Increasing Access to Trauma Focused Cognitive Behavioural Therapy for Post Traumatic Stress Disorder Through a Pilot Feasibility Study of a Group Clinical Supervision Model
}

\author{
Neil J. Kitchiner, Bethan Phillips, Neil Roberts and Jonathan I. Bisson \\ Cardiff and Vale NHS Trust, Dept of Traumatic Stress Service, University Hospital of Wales, Cardiff, UK
}

\begin{abstract}
Trauma focused cognitive behavioural therapy (TFCBT) is recommended as a first line treatment for post traumatic stress disorder (PTSD). Unfortunately, it is not widely available, often resulting in long waits for sufferers. We attempted to overcome this through a pilot feasibility study of brief training and supervision with a group of mental health professionals (MHPs). The MHPs attended a structured weekly clinical supervision group adhering to a cognitive therapy model of supervision. Eleven PTSD sufferers were treated during the pilot phase. Davidson Trauma Scale scores dropped by a mean of 36.5 points ( $95 \%$ C.I. 12.8, 60.5) over the course of treatment. Group Clinical Supervision for TFCBT appears to have the potential to offer a clinically and cost-effective model of maximizing treatment availability for PTSD sufferers.
\end{abstract}

Keywords: PTSD, traumatic stress, group supervision, cognitive behaviour therapy, clinical supervision.

\section{Introduction}

Posttraumatic stress disorder (PTSD) is a common condition with a lifetime prevalence of approximately $7 \%$ in the general population (Kessler, Sonnega, Bromet, Hughes and Nelson, 1995). It can cause significant distress and suffering. The National Institute of Clinical Excellence (NICE) Guidelines on PTSD (NICE, 2005) recommend that all PTSD sufferers be offered interventions with a trauma focused psychological treatment (TFCBT or Eye Movement Desensitization and Reprocessing, EMDR). Unfortunately, despite the existence of potentially effective treatments there are often insufficient resources to meet the needs of PTSD sufferers. There have been few attempts to evaluate the effectiveness of a training and supervision programme of this kind for trainee therapists.

\section{Aim}

To evaluate the introduction of a TFCBT educational training package and subsequent group clinical supervision, with MHPs with no prior TFCBT training, in the treatment of those suffering from PTSD.

Reprint requests to Neil J. Kitchiner, Cognitive-Behavioural Psychotherapist, Department of Liaison Psychiatry \& Traumatic Stress Service, Cardiff and Vale NHS Trust, University Hospital of Wales, Heath Park, Cardiff, South Wales, CF14 4XW. E-mail: neil.kitchiner@cardiffandvale.wales.nhs.uk 
Group clinical supervision (GCS). Many clinical supervision groups are unstructured, with discussion of case issues occurring in a non-systematic manner. Liese and Beck (1997) attempted to overcome this by developing a model based on the process of individual cognitive therapy, which is structured, focused and educational. Their model places a strong emphasis on collaboration and agenda setting with supervisees for session structure and content. Group clinical supervision tends to be more didactic than individual sessions, focusing more on common problems or themes rather than individual therapists' difficulties. Liese, Barber and Beck (1995) advocated a GCS approach where trainees can directly observe an expert conducting cognitive therapy, followed by discussion of the therapy process observed. This model of supervision aims to teach cognitive therapy theory and techniques, to correct misconceptions of cognitive therapy, and to reduce the likelihood of therapist drift. We felt that Liese and Beck's (1997) model could be adapted for use in our clinical setting and therefore based the development of our TFCBT supervision group on it.

\section{Method}

Two supervision groups for five MHPs were created. Group members required previous experience of psychiatry and/or counselling but did not require any qualification or specialist knowledge in CBT or traumatic stress. The groups met weekly and were facilitated by a cognitive behavioural psychotherapist (NJK).

The first six group sessions were dedicated to training the MHPs to deliver a specific form of TFCBT, via education, case discussions, role-play, and training in manualized prolonged exposure (Foa, Dancu and Hembree, 2002). Once the 10 MHPs and group supervisor felt they were ready to use the TFCBT skills they began to treat individuals who had been assessed by the group's supervisor as suffering from uncomplicated DSM-IV PTSD. Therapy sessions were on a weekly basis. Self report clinical measures were used to determine outcome. MHPs were contracted to provide an audiotape every five sessions for delayed supervision. Taping of sessions remains one of the most valuable features of cognitive therapy supervision and minimizes the often biased views of self-report from the supervisee.

In order to evaluate the effectiveness of the MHPs use of TFCBT, the results of the clinical measures were analysed using SPSS-10. Paired sample $t$-tests were used to explore changes between pre- and post-treatment continuous data. The number of supervision sessions attended by each group member was recorded, as was the number and length of sessions with each client.

\section{Results}

Four nurses, one midwife, four junior psychiatrists, and one psychologist were members of the group. The junior psychiatrists had worked for 6-24 months in psychiatry, the four registered mental health nurses had worked for 4-30 years in psychiatry, the midwife had no mental health experience (but a diploma in counselling) and the psychologist had a research background.

At the time of the evaluation of this pilot there were 10 group members who attended between 6 and 23 supervision sessions (mean $=16.3, S D=5.8$ ). Each group member treated one patient for between 5 and 16 sessions (mean $=9.8, S D=3.7$ ). The total time spent with each patient was between 4 and 19 hours (mean $=10.0, S D=4.7)$. All patients completed their course of TFCBT. 
Table 1. Pre to post-treatment comparison of outcome measures

\begin{tabular}{lccccc}
\hline Measure & $N$ & $\begin{array}{c}\text { Pre-treatment } \\
\text { mean }(S D)\end{array}$ & $\begin{array}{c}\text { Post-treatment } \\
\text { mean }(S D)\end{array}$ & $\begin{array}{c}\text { Mean difference } \\
(95 \% \mathrm{CI})\end{array}$ & $p$ value \\
\hline BDI & 11 & $29.8(14.2)$ & $19.8(13.5)$ & $10.0(4.9,15.1)$ & .001 \\
DTS & 11 & $89.1(23.5)$ & $52.5(33.1)$ & $36.5(12.8,60.5)$ & .007 \\
LAS - Home & 9 & $3.8(2.7)$ & $2.7(2.8)$ & $1.1(-0.8,3.0)$ & .21 \\
LAS - Work & 9 & $5.1(2.9)$ & $2.9(3.0)$ & $2.2(0.6,3.8)$ & .01 \\
LAS - Private leisure & 9 & $5.1(2.3)$ & $2.9(2.3)$ & $2.2(2.5,0.9)$ & .03 \\
LAS - Social leisure & 9 & $5.3(2.0)$ & $4.0(2.5)$ & $1.3(2.2,0.8)$ & .11 \\
LAS - Relationships & 9 & $4.2(2.5)$ & $2.4(1.7)$ & $1.8(-0.9,4.5)$ & .17 \\
\hline
\end{tabular}

Note: $\mathrm{BDI}=$ Beck Depression Inventory, a 21 -item questionnaire that rates how the individual has been feeling over the past 2-weeks, including today, from a selection of four possibilities. DTS = Davidson Trauma Scale, a 17-item self-report measure that rates the individuals specific DSM-IV PTSD symptoms on two ratings, Frequency and Severity using a $0-4$ scale. LAS = Life Adjustment Scale, the individual rates how the problem affects work, home management, social leisure, private leisure and relationships using a $0-8$ scale where $0=$ no problem and $8=$ severely affected.

Sixteen patients were offered TFCBT with a trainee. All 16 met diagnostic criteria for chronic PTSD. Four patients dropped out of therapy and one was not suitable due to a lack of traumatic stress symptoms when invited to start therapy. Table 1 shows a comparison of preand post-treatment scores using the paired samples $t$-test clinical outcome measures for the 11 patients who completed a course of TFCBT. Significant differences were found between pre and post scores for the Beck Depression Inventory (BDI), Davidson Trauma Scale (DTS), and two of the Life Adjustment Scales. None of the 11 patients who completed therapy continued to meet the criteria for PTSD post treatment.

\section{Limitations}

This feasibility study has some limitations, including the absence of a control group and the small numbers included in the study. It is therefore important that we remain guarded regarding the effectiveness of the brief training and supervision group. It could be argued that the changes that took place were a result of naturalistic improvement rather than a result of psychological intervention (Kessler et al., 1995), although all the patients treated had chronic PTSD and significant spontaneous improvements were therefore less likely. There were also some treatment drop-outs and it is uncertain whether the relative inexperience of the therapists played any part in this. A possible next step in evaluating this model would be to design a manualized educational training package and evaluate the two-component educational intervention. This could be done via a double-baseline or randomized controlled design.

\section{Clinical implications}

This pilot study appears to demonstrate the potential of a GCS model in training and supervision of MHPs to develop knowledge and skills in TFCBT. A consequence of the recent publication of the NICE Guidelines for PTSD (2005) will be an increased awareness of the existence of PTSD and evidence-based treatments. This is likely to lead to an increased demand for trauma 
focused psychological treatments, including TFCBT. The GCS described in this paper could be one way to address the currently limited availability of such treatment and support the development of more comprehensive services in the future.

\section{References}

Foa, E. B., Dancu, C. V. and Hembree, E. (2002). Manualised Treatment for Prolonged Exposure in Treating Posttraumatic Stress Disorder. International Traumatic Stress Studies Conference (Workshop), Baltimore, Maryland, USA.

Kessler, R. C., Sonnega, A., Bromet, E., Hughes, M. and Nelson, C. B. (1995). Posttraumatic stress disorder in the National Comorbidity Survey. Archives of General Psychiatry, 52, 1048-1060.

Liese, B. S., Barber, J. and Beck, A. T. (1995). The Cognitive Therapy Adherence and Competence Scale. Unpublished instrument, University of Kansas Medical Centre, Kansas City.

Liese, B. and Beck, J. S. (1997). Cognitive therapy supervision. In C. E. Watkins (Ed.), Handbook of Psychotherapy Supervision. New York: Wiley.

NICE (2005). Post-Traumatic Stress Disorder: The management of PTSD in adults and children in primary and secondary care. National Clinical Practice Guideline Number 26. London: Gaskell and the British Psychological Society. 